\title{
Vaccinating People with Obesity for COVID-19: EASO Call for Action
}

\author{
Dror Dicker $^{a, b, c}$ Rachel Golan ${ }^{d}$ Jennifer Lyn Baker ${ }^{a, e} \quad$ Luca Busetto $^{a, f}$ \\ Gema Frühbeck $^{\mathrm{a}, \mathrm{g}}$ Gijs H. Goossens ${ }^{\mathrm{a}, \mathrm{h}}$ Jason C.G. Halford ${ }^{\mathrm{a}, \mathrm{i}}$ \\ Jens-Christian Holm ${ }^{a, j}$ Euan Woodward ${ }^{a}$ Nathalie J. Farpour-Lambert ${ }^{a, k}$ \\ ${ }^{a}$ European Association for the Study of Obesity (EASO), Teddington, UK; ${ }^{b}$ Department of Internal Medicine D, \\ Hasharon Hospital, Rabin Medical Center, Petah Tikva, Israel; ' $S a c k l e r$ School of Medicine, Tel Aviv University, Tel \\ Aviv, Israel; ${ }^{d}$ Department of Public Health, Ben-Gurion University of the Negev, Beer Sheva, Israel; ${ }^{\text {CCenter for }}$ \\ Clinical Research and Prevention, Bispebjerg and Frederiksberg Hospital, Frederiksberg, Denmark; ${ }^{\mathrm{f}}$ Department of \\ Medicine, University of Padova, Padova, Italy; ${ }^{9}$ Department of Endocrinology \& Nutrition, Clínica Universidad de \\ Navarra, University of Navarra, IdiSNA, CIBEROBN, Spanish Health Institute Carlos III, Pamplona, Spain; ${ }^{\mathrm{h}}$ Department \\ of Human Biology, NUTRIM School of Nutrition and Translational Research in Metabolism, Maastricht University \\ Medical Centre, Maastricht, The Netherlands; iSchool of Psychology, University of Leeds, Leeds, UK; 'The Children's \\ Obesity Clinic, Department of Paediatrics, Holbæk University Hospital, Holbæk, Denmark; ${ }^{\circ}$ Obesity Prevention and \\ Care Program, Service of Endocrinology, Diabetology, Nutrition and Therapeutic Patient Education, Department of \\ Medicine, University Hospitals of Geneva, Geneva, Switzerland
}

\section{Keywords}

COVID-19 - Obesity $\cdot$ Vaccination

The European Association for the Study of Obesity (EASO), in collaboration with the EASO European Coalition for People Living with Obesity (ECPO), is committed to sharing knowledge, evidence-based recommendations, and guidance toward the clinical, patient, research, and policy communities. Since the beginning of the coronavirus disease 2019 (COVID-19) pandemic, our mission has been to identify the particular needs of individuals living with obesity, healthcare providers, and policy makers [1].

Obesity, a complex chronic disease in which abnormal or excessive fat impairs health, is associated with increased risks for the initiation, progression, and outcomes of COVID-19 [2]. Adults living with obesity are more likely to require acute care and admission to the intensive care unit, intubation, and mechanical ventilation, especially among those younger than 60 years [2-5]. Mortality from COVID-19 was found to be higher among pa-

karger@karger.com www.karger.com/ofa

Karger"
(C) 2021 The Author(s)

Published by S. Karger AG, Basel

This is an Open Access article licensed under the Creative Commons Attribution-NonCommercial-4.0 International License (CC BY-NC) (http://www.karger.com/Services/OpenAccessLicense), applicable to the online version of the article only. Usage and distribution for commercial purposes requires written permission. tients with obesity in some studies $[4,5]$. Obesity is also the most commonly reported underlying medical condition in US children presenting severe COVID-19 [6].

People living with obesity often have a systemic lowgrade inflammation, a higher susceptibility to infections, and a dampened immune response to infectious agents [7]. Obesity may also play a role in altering the viral life cycle, together with the intrinsic immunological perturbations and associated impaired immune response, leading to more severe clinical outcomes as compared to individuals with normal body mass index (BMI). The prolonged viral clearance demonstrated in people with obesity infected with SARS-CoV-2 may support the relation between obesity and the risk of severe COVID-19. The positive correlation between body weight and time to negative detection of COVID-19 genes might suggest a larger viral load in people with obesity, while angiotensinconverting enzyme 2 in adipose tissue might be a critical link between obesity, non-communicable diseases, and the susceptibility to and severity of COVID-19 [8]. 
People living with obesity demonstrate an impaired immune response to influenza A virus (IAV) vaccination and antimicrobial treatment [9]. Hence, the efficacy of other vaccines might be reduced in this population. Although data regarding the effectiveness of vaccination for COVID-19 in people with obesity is unavailable, it is known from studies regarding vaccination for IAV H1N1 that host-associated factors such as obesity can reduce vaccine efficacy [10]. Higher BMI was associated with a greater decline in influenza A antibody titers after 12 months, suggesting that obesity may impair the ability to mount a protective immune response to influenza virus [11]. The relatively lower effectiveness of influenza vaccines in people with obesity is hypothesized to be mediated by insufficient $\mathrm{T}$ cell function, since peripheral blood mononuclear cells from vaccinated adults with obesity show decreased activation of cytotoxic $\mathrm{T}$ cells and reduced expression of functional markers [10].

Subgroup analysis in COVID-19 vaccination trials demonstrated that the efficacy among adults with obesity was, in the short term, generally consistent with that observed in the overall population [12]. A recent randomized trial, which estimated vaccine efficacy in the shortterm, for patients with obesity and other coexisting conditions such as hypertension found effectiveness to be slightly lower among those with higher numbers of coexisting conditions [13]. Therefore, we conclude that people living with obesity should be encouraged to be vaccinated against COVID-19, and prioritized, similar to oth- er populations at risk of severe outcomes due to preconditions, such as diabetes mellitus, chronic cardiovascular disease or respiratory disease, immunocompromised states, or cancers. In children, ongoing studies are currently investigating the efficacy and safety of COVID-19 vaccines. Accordingly, we are calling for:

- Considering people living with obesity as at-risk of severe COVID-19;

- Prioritizing people with obesity for COVID-19 vaccination;

- Promoting studies that will examine the long-term efficacy of COVID-19 vaccination on neutralizing antibody levels and the infection rate in children, adolescents, and adults with obesity.

\section{Conflict of Interest Statement}

The authors of this manuscript do not have any conflicts of interest to declare.

\section{Funding Sources}

This manuscript did not receive any funding.

\section{Author Contributions}

Working on behalf of EASO, all authors contributed jointly to the formulation and writing of this position statement.

\section{References}

1 Frühbeck G, Baker JL, Busetto L, Dicker D, Goossens GH, Halford JC, et al. European Association for the Study of Obesity Position Statement on the Global COVID-19 Pandemic. Obes Facts. 2020;13(2):292-6.

2 Goossens GH, Dicker D, Farpour-Lambert NJ, Frühbeck G, Mullerova D, Woodward E, et al. Obesity and COVID-19: a perspective from the European Association for the Study of Obesity on immunological perturbations, therapeutic challenges, and opportunities in obesity. Obes Facts. 2020;13(4):439-52.

3 Busetto L, Bettini S, Fabris R, Serra R, Dal Pra C, Maffei P, et al. Obesity and COVID-19: an Italian Snapshot. Obesity. 2020 Sep;28(9):1600-5.

4 Cariou B, Hadjadj S, Wargny M, Pichelin M, Al-Salameh A, Allix I, et al.; CORONADO investigators. Phenotypic characteristics and prognosis of inpatients with COVID-19 and diabetes: the CORONADO study. Diabetologia. 2020 Aug;63(8):1500-15.

Vaccinating People with Obesity for COVID-19
5 Simonnet A, Chetboun M, Poissy J, Raverdy V, Noulette J, Duhamel A, et al.; LICORN and the Lille COVID-19 and Obesity Study Group. High prevalence of obesity in severe acute respiratory syndrome coronavirus-2 (SARS-CoV-2) requiring invasive mechanical ventilation. Obesity. 2020 Jul;28(7):1195-9.

6 Kim L, Whitaker M, O'Halloran A, Kambhampati A, Chai SJ, Reingold A, et al; COVID-NET Surveillance Team. Hospitalization rates and characteristics of children aged $<18$ years hospitalized with laboratory-confirmed COVID-19 - COVID-NET, 14 States, March 1-July 25, 2020. MMWR Morb Mortal Wkly Rep. 2020;69(32):1081-1088.

7 Luzi L, Radaelli MG. Influenza and obesity: its odd relationship and the lessons for COVID-19 pandemic. Acta Diabetol. 2020 Jun;57(6):759-64.

8 Dicker D, Lev S, Gottesman T, Kournos T, Dotan M, Ashorov N, et al. A Time Frame for Testing Negative for SARS-COV2 in People with Obesity. Obes Facts. 2020;13(5):528-33.
9 Honce R, Schultz-Cherry S. Impact of obesity on influenza A virus pathogenesis, immune response, and evolution. Front Immunol. 2019 May;10:1071.

10 Dhakal S, Klein SL. Host factors impact vaccine efficacy: implications for seasonal and universal influenza vaccine programs. J Virol. 2019 Oct;93(21):e00797-19.

11 Sheridan PA, Paich HA, Handy J, Karlsson EA, Hudgens MG, Sammon AB, et al. Obesity is associated with impaired immune response to influenza vaccination in humans. Int $J$ Obes. 2012 Aug;36(8):1072-7.

12 Polack FP, Thomas SJ, Kitchin N, Absalon J, Gurtman A, Lockhart S, et al.; C4591001 Clinical Trial Group. Safety and Efficacy of the BNT162b2 mRNA Covid-19 Vaccine. N Engl J Med. 2020 Dec;383(27):2603-15.

13 Dagan N, Barda N, Kepten E, Miron O, Perchik S, Katz MA, et al. BNT162b2 mRNA Covid-19 Vaccine in a Nationwide Mass Vaccination Setting. N Engl J Med. 2021 Feb;384(15):1412-23.

COVID-19 